\title{
APROXIMACIÓN A LA EVOLUCIÓN RECIENTE DE LA GRAN PROPIEDAD AGRÍCOLA EN EL BAJO SEGURA: EL CASO DE ORIHUELA
}

\author{
Gregorio Canales Martínez y Fermín Crespo Rodríguez
}

\section{RESUMEN}

El dilatado término municipal de Orihuela, en su mayoría de secano, ha motivado la permanencia de la gran propiedad agrícola hasta nuestros días. En el artículo se estudia la evolución reciente de estas grandes haciendas desde los años cuarenta a la actualidad. Resulta interesante el análisis de estos predios por cuanto el secano del Bajo Segura ha experimentado en estas últimas décadas un cambio trascendental, debido, por un lado, a la transformación y puesta en riego de los terrenos con la llegada del Trasvase Tajo-Segura, y de otro, al hecho de que, el extenderse por la zona litoral, ello ha generado la creación de urbanizaciones. Por consiguiente, el municipio de Orihuela conoce un importante proceso de cambio del uso del suelo, que ha pasado de una dedicación agrícola de bajo rendimiento a otra más especulativa, centrada en el uso residencial-turístico y en la implantación de una economía de mercado. No hay una relación excluyente entre los dos factores, ya que se observa en algunas fincas una compaginación de ambos aprovechamientos.

\begin{abstract}
The municipality of Orihuela has a huge extension of land, most of it unirrigated. This is the reason why the study analyses the recent evolution of these large properties from the decade of the forties onwards. These country estates of the Bajo Segura have experimented in the last decades changes of great significance, on one hand, the dry lands have been transformed into irrigated ones with the waters of the Trasvase Tajo-Segura; on the other hand, and due to the fact that they are close to the coast, some of them are now turistic urbanizations. Therefore, we now find a speculative use of the land based on a fertile agriculture and on a turistic development. One use does not exclude the other and in some properties both of them are combined.
\end{abstract}


La evolución de las grandes propiedades en el término municipal de Orihuela responde a unos acontecimientos históricos que han hecho pervivir hasta nuestros días su considerable tamaño. Se pretende ahora realizar una aproximación al conocimiento de dicha evolución en el período de tiempo comprendido entre principios de los años cuarenta y la actualidad.

Resulta interesante analizar la evolución de estas fincas por cuanto, al estar ubicadas en el secano, el cambio experimentado en estos últimos años es trascendental, debido, por un lado, a la transformación y puesta en riego de los terrenos con la llegada del trasvase Tajo-Segura, y de otro al hecho de que al extenderse por la zona litoral, ello ha generado la creación de modernas urbanizaciones y zonas residenciales, compaginándose el aprovechamiento tradicional agrícola con el turístico de reciente implantación.

El hecho que nos decidió a tomar como referencia para el estudio el municipio de Orihuela, además de lo anteriormente expuesto, es su gran tamaño, unos $443 \mathrm{Km}^{2}$, que representan el 46,5\% de la superficie total del Bajo Segura.

El que los datos estén referidos única y exclusivamente al tiempo transcurrido desde finales de la Guerra Civil hasta nuestros días, se debe a que la fuente de investigación es el Registro de la Propiedad, cuyo material desapareció en un incendio durante dicho conflicto, reconstruyéndose con posterioridad. Por ello sólo se ha podido realizar un esbozo de la evolución de estas grandes fincas en momentos anteriores a dicha guerra.

\section{Antecedentes históricos}

Durante el siglo XIX estas grandes haciendas pertenecían a religiosos y nobles. La desamortización de las propiedades eclesiásticas representó para estos últimos la posibilidad de ampliar considerablemente sus patrimonios, que en algunos casos conservaron hasta bien entrada la actual centuria y no será hasta los años cuarenta, cuando se inicie el proceso de venta.

Los beneficios que concedía la Ley de Colonias Agrícolas de 3 de junio de 1868, determinaron que algunos de estas grandes haciendas se roturaran, y en general que en todas ellas se efectuaran nuevas plantaciones, en las que dominan los cultivos arbóreos. A la vez, con la aplicación de la Ley, se consigue la dispersión de la población mediante la creación de casas de labranza (1).

Los convenios jurídicos que regulan estos asentamientos reflejan la intensa actividad colonizadora que se va a ejercer en el campo. Las cláusulas de los contratos ponen de manifiesto detalladamente como se debía realizar la puesta en 
cultivo. Generalmente se trata de un acuerdo entre propietario y colono por el cual este último, se obligaba a plantar cada año una determinada superficie de la finca con el cultivo que le era señalado, corriendo siempre él con los gastos. El contrato más usual era la aparcería, que se efectúa mediante la partición de la cosecha, y que solía ser de la mitad para el arbolado, mientras que para el sembrado variaba la proporción, siendo la mayor parte para el aparcero. Aun así, no se excluye el arrendamiento como forma contractual.

Estos regímenes de tenencia perviven hasta después de la Guerra Civil, si bien desde entonces, conforme se abren expectativas de mercado y se supera la etapa de autarquía, los cultivos de estas grandes haciendas de secano pierden rentabilidad, siendo por ello abandonadas en muchas ocasiones.

La transformación al regadío de estos terrazgos se va a acometer cuando los dueños de las fincas se apoyen en las nuevas técnicas para la extracción de agua y aprovechen los acuíferos de la zona. Las elevaciones dieron en un primer momento calidades muy óptimas, pero por la mayor presión sobre el manto hipogeo se salinizan los caudales, a la vez que se produce un aumento progresivo de precios por la mayor profundidad a la que se realizan los bombeos, debido a la disminución del nivel piezométrico.

El factor principal que va a condicionar la transformación de estas grandes propiedades de secano fue la consideración de esta zona como beneficiaria de las aguas del trasvase Tajo-Segura, lo que provocó que sus titulares, en un alto porcentaje todavía absentistas, se sintieran atraídos por la tierra, tanto para su explotación directa como para la parcelación. La concesión de futuros caudales a trasvasar generó en toda la zona grandes inversiones ante las expectativas de la llegada de estas aguas, que se manifiesta en la construcción de toda la infraestructura de riego y viaria necesaria, para la puesta en funcionamiento de las explotaciones, así como el acondicionamiento del terrazgo, habiendo en ocasiones que recurrir a la creación de abancalamientos en terrazas para conseguir superficies aptas para el cultivo. Obras estas fuera del alcance de los pequeños propietarios y arrendatarios o aparceros. Esta falta de capital también afectaba a veces a los grandes propietarios y por ello, dadas las excepcionales características para el cultivo y el turismo que posee la comarca, va a ser centro de acción directa de las grandes empresas que pueden sufragar los gastos para la puesta en cultivo o urbanización de los terrenos.

\section{Evolución reciente de las grandes haciendas}

El estudio actual de las grandes haciendas se ha realizado mediante un muestreo en el Registro de la Propiedad de Orihuela. Con los datos obtenidos hemos confeccionado el cuadro 1, en el que se recoge el nombre de la finca, su superficie y ubicación en el término municipal. De todas las fincas estudiadas, solamente Vista Alegre no llega a 100 Ha., cinco de ellas pasan de 500 Ha. y de estas últimas, Campoamor se aproxima a $2.000 \mathrm{Ha}$. 


\section{CUADRO 1}

Relación de las fincas analizadas

$\begin{array}{llrl}\text { Nombre de la finca } & & \text { Superficie (Ha.) } & \text { Partido rural } \\ \text { Campoamor } & (2) & 1.984 .3680 & \text { La Horadada } \\ \text { Ballesta de Arriba } & (3) & 729.3311 & \text { Rebate } \\ \text { Lo Romero } & (4) & 705.7106 & \text { La Horadada } \\ \text { El Espartal } & (5) & 651.2813 & \text { Campo de Salinas } \\ \text { Dehesa de Roca } & (6) & 516.3449 & \text { Cartagena } \\ \text { Las Colinas } & (7) & 458.2895 & \text { Campo de Salinas } \\ \text { Lo Monte } & (8) & 355.5890 & \text { La Horadada } \\ \text { Bancal de don Luis } & (9) & 306.8128 & \text { Campo de Salinas } \\ \text { Lo Gracia de Arriba } & (10) & 254.3897 & \text { Rebate } \\ \text { Las Animas } & (11) & 240.9830 & \text { La Murada } \\ \text { El Estrecho } & (12) & 225.2714 & \text { Campo de Salinas } \\ \text { La Asomada } & (13) & 197.9429 & \text { Campo de Salinas } \\ \text { Lo Sastre y Soler } & (14) & 142.2254 & \text { Campo de Salinas } \\ \text { La Regia } & (15) & 114.9421 & \text { Los Dolces } \\ \text { La Cenia } & (16) & 112.5701 & \text { Campo de Salinas } \\ \text { Vista Alegre } & (17) & 81.3029 & \text { La Murada }\end{array}$

Fuente: Registro de la Propiedad de Orihuela. Libro de inscripciones del término municipal de Orihuela: (2) tomo 38, folio 147; (3) t. 234, f. 183; (4) t. 106, f. 93; (5) t. 54, f. 18; (6) t. 688, f. 1; (7) t. 243 , f. 104 , (8) t. 166 , f. 87 ; (9) t. 255 , f. 233 ; (10) t. 493 , f. 219 ; (11) t. 119 , f. 172 ; (12) t. 265 , f. 222 ; (13) t. 181 , f. 88 ; (14) t. 15 , f. 164 ; (15) t. 254 , f. 76 ; (16) t. 189 , f. 41 ; (17) t. 15 , f. 182 .

Las fincas se ubican en el secano tradicional (Campo de Salinas, Murada, Horadada, Rebate, Los Dolces), lugares donde en los últimos años, y debido a las aportaciones del trasvase Tajo-Segura, se han producido grandes transformaciones agrícolas que han ampliado enormemente el espacio cultivado, el cual se encontraba, en el mejor de los casos, con los aprovechamientos más característicos del secano.

Por el contrario otras fincas no han sido transformadas, puesto que la tenencia de la tierra se dirigía a la obtención de prestigio social. Tal es el caso de «Peña del Águila», la cual ha sido vendida reiteradas veces, sin observarse modificación alguna de su terrazgo: «terreno secano en su mayor parte lomas incultas que producen pastos y leñas de monte bajo y algunos pinos, cuyas lomas sirven de vertientes a pequeñas extensiones de cultivo que forman las cañadas, en las 
que arraigan algunos árboles entre ellos algarrobos, almendros, higueras, y olivos, también una casa de labor y contigua a la misma un pozo». Todo ello con una extensión de 129 Ha. y que en 1983 fueron adquiridas por un industrial ilicitano.

Se detecta la pervivencia de la titularidad del estamento nobiliario en algunas de estas haciendas hasta nuestros días, este es el caso de las fincas «El Estrecho» y «La Asomada», que pertenecían al duque de Pino Hermoso y que fueron adquiridas por un comerciante de Orihuela. O como la hacienda «Lo Sastre y Soler», que en 1975 pasó de la familia Roca de Togores a un industrial ilicitano.

La puesta en explotación de algunas de estas haciendas llevó consigo un creciente endeudamiento por parte de sus propietarios, hasta el extremo de que algunos perdieron la titularidad del terreno a manos de las instituciones financieras, este es el caso de «La Cenia»y de «Cueva Sierra». La topografía del terreno y las dificultades que suponía la obtención de caudales, hicieron necesarias inversiones cuantiosas, recurriendo habitualmente a préstamos bancarios, que en algunos casos resultaron de difícil amortización, por lo que se llegaba a la hipoteca del terreno.

Las transformaciones en regadío empiezan en el mejor de los casos a principios de los años cuarenta, como es el caso de la finca «El Espartal». Dicha finca situada en el partido de Salinas y con una extensión de 651 Ha. fue adquirida en 1941 por un abogado de San Javier, quien la permuta años después por otras fincas y bienes semovientes a un vecino de Yeste. Este lleva a cabo los inicios de transformación en regadío, realizando sondeos en busca del manto hipogeo que proporcionaron unos caudales de 15 y 20 1/s en los pozos abiertos. A partir de entonces se hicieron varios proyectos de obras de desfonde, despedregado, abancalamiento y nivelación de la parte de terreno que permanecía improductiva.

Además de los alumbramientos de agua realizados individualmente, era usual en las transformaciones de secano acometer las extracciones entre varias personas repartiendo gastos y beneficios. Así, esta finca se beneficiaba del 50\% del caudal alumbrado en una hacienda vecina, teniendo derecho uno de los propietarios a ampliar el alumbramiento del pozo existente mediante nuevas galerías o perforaciones. De los socios en la extracción, el que no tenía la iniciativa de ampliar, solamente tenía derecho a doblar su caudal, el otro se quedaba con el resto, que incluso podía vender a terceros.

Otras veces la puesta en riego depende de la creación de una sociedad, tal es el caso de la hacienda «Bancal de Don Luis», para cuya transformación se constituyó en 1961 una sociedad mercantil anónima llamada: Agrícola Industrial Levantina S. A., con 200 acciones, de las que el titular de la finca posee 196 y las 4 restantes se las reparten por igual un industrial y un comerciante de Madrid. El objeto de la misma es la explotación agrícola.

Dicha sociedad se declaró en suspensión de pagos en 1966 y su Junta General 
acordó enajenar la totalidad de los bienes de su activo para pagar a sus acreedores en un plazo máximo de tres años. Para ejecutar dicha venta se crea una comisión que traspasó en ese mismo año la hacienda a la sociedad mercantil anónima Playa Flamenca S. A., constituida en 1960 y con alto porcentaje de capital belga. Esta empresa ha llevado a cabo la transformación del terrazgo, derivando su primitiva utilización agrícola en turística.

No es un caso aislado la constitución de sociedades que inviertan en estas fincas por las grandes posibilidades de ganancias que ofrecen. Esto se observa, además de en la anteriormente citada sociedad Playa Flamenca S. A., en la hacienda «Lo Sastre y Soler», que en unión de otras («las Colinas» y «Cueva del Pastor») reúnen una superficie de 592 Ha. y que en 1979 pasan a MONTEAGRO S. A. También en «El Estrecho»y en «La Asomada» hay segregaciones a favor de la empresa Agrícola del Segura S. A. (AGRISESA) de 174 Ha. en 1973. Por último, en «El Espartal», la Caja Rural Cooperativa Agrícola de Orihuela adquirió 302 Ha. en 1972.

\section{Diversificación de los aprovechamientos de las grandes haciendas}

Podemos determinar tres líneas diferentes en la evolución de estas fincas:

- Las que compaginan el aprovechamiento agrícola y turístico, que son fincas próximas o colindantes a la línea de la costa, por lo cual en los últimos años, y debido al «boom» turístico, han sido objeto de una gran demanda para parcelación y aprovechamiento residencial, manteniendo hacia el interior la utilidad agrícola con nuevos cultivos que han aumentado su rentabilidad.

- Las que tienen aprovechamiento agro-industrial, son aquellas haciendas que han pasado a manos de grupos financieros o industriales y en las que se han producido importantes transformaciones en el paisaje, tendentes a crear un sistema productivo de tipo comercial mediante la introducción del regadío a gran escala.

- Las que se fragmentan en pequeños lotes de aprovechamiento exclusivamente agrícola, que son las fincas que han sido objeto de venta por sus propietarios en lotes de todo tamaño a agricultores o pequeños inversores, que buscan ganancias en las nuevas expectativas económicas del campo.

3-a. Fincas agro-turísticas: Como fincas más características de la zona dentro del aprovechamiento agro-turístico debemos resaltar «Campoamor» y «La Cenia».

La finca «Campoamor» representa un ejemplo en cuanto a transformación agrícola desde principios del siglo XVIII hasta nuestros días. Esta hacienda de secano situada en el partido de La Horadada, con una superficie de 1.984 Ha., importante proceso colonizador que arranca de principios del siglo 
XVIII, cuando el dominio útil de este predio se transfiere, por el Ayuntamiento de Orihuela, al convento de La Merced, que va a iniciar la roturación mediante el asentamiento de colonos a censo enfitéutico, explotación que no culminó con el éxito deseado (18).

Tras el proceso desamortizador del siglo XIX la finca fue adquirida por el escritor D. Ramón de Campoamor, quien para su explotación se acogió a los beneficios que las diversas leyes de colonias agrícolas concedían durante la segunda mitad del siglo XIX, fruto de esta actividad roturadora aparecen varias casas de labor dispersas dentro del predio.

En 1920 la finca fue vendida por uno de los descendientes de Campoamor a unos ganaderos de San Miguel de Salinas. Años después (1941) también por venta llega a manos de D. Antonio Tarragas Escribano, industrial de San Pedro del Pinatar, en sociedad con su cuñado. Es a partir de entonces cuando se produce un auténtico proceso de cambio que empieza a sustituir los antiguos aprovechamientos de secano compaginados con aprovechamientos ganaderos, por otros cultivos más rentables mediante la explotación de los acuíferos y la transformación de aquellas zonas más favorables en regadío.

A principios de los años sesenta se inicia el proceso de urbanización de dicha hacienda, uniéndose al tradicional carácter agrícola el aprovechamiento turístico, quedando configurada como una explotación típica agro-turística, aspecto este que en los años siguientes se va a ir revalorizando dado el incremento de la zona residencial, junto a una sobrevalorización de la zona agrícola, al beneficiarse de las aguas del trasvase a principios de la década actual y con la introducción de los cultivos forzados bajo plástico. Completándose en este último momento la transformación de montes en feraces huertos de cítricos (19).

La actividad turística se centra en la zona de costa de la explotación, en un proceso de venta de lotes que se inicia en 1959 y llega hasta 1981. En total son 164 el número de lotes vendidos, que comprenden una superficie de 1.071 Ha. lo que representa la enajenación de algo más de la mitad de la explotación $(53,9 \%)$.

La hacienda «La Cenia» está situada en Campo de Salinas, tiene una superficie de 112 Ha., y al igual que la anterior llega hasta la línea de costa. Hasta los años 40 tiene una dedicación exclusiva de agricultura extensiva de secano. A partir de esa fecha se inicia el proceso de puesta en riego mediante la perforación de pozos (uno de ellos proporcionó un caudal de 40 1/s) además cuenta con cuatro balsas reguladoras de las que parte una red de canales de distribución de agua con una longitud de $7 \mathrm{Km}$.

La finca pertenecía en esos años a un agricultor de San Fulgencio, que fue el encargado de acometer las obras de regadío. La falta de pago de los créditos concedidos para la transformación del terreno, motivó que en 1952 se decretara la venta en pública subasta de dicha explotación, celebrada sin ningún licitador el 30 de mayo, con posterioridad se realizó una segunda que fue adjudicada al 
Banco Hipotecario de España en 1956, dicho banco la vende a los hijos de un industrial madrileño y a partir de aquí se suceden las ventas entre personas domiciliadas en Madrid, hasta que en 1958 la compra un comerciante vecino de San Sebastián, el cual se decide a iniciar su urbanización, denominándola «La Zenia». El proceso de venta de los lotes se inicia en 1964 y llega hasta la actualidad. Se han segregado un total de 323 lotes, con una superficie de 27,4606 Ha. que representan una mínima parte del predio $(24,4 \%)$.

Es de destacar el predominio que en La Cenia tienen los lotes de tamaño pequeño y mediano (entre 600 y $900 \mathrm{~m}^{2}$ ) que corresponde a una dedicación básicamente residencial, mientras que en Campoamor, además de los tamaños más reducidos de orientación turística, aparecen seis compradores de gran tamaño, el 98,48\% del total vendido, que corresponde a particulares y a empresas dedicadas a la construcción o a la producción agrícola (cuadro II).

\section{CUADRO 2}

Clasificación de los compradores de tierra en la finca-urbanización Campoamor según la superficie total adquirida (1959-1981)

\begin{tabular}{|c|c|c|c|c|}
\hline Superficie $\mathrm{m}^{2}$ & Compradores & $\%$ & Total $\mathrm{m}^{2}$ & $\%$ \\
\hline-299 & 2 & 1.48 & 337 & 0,01 \\
\hline $300-\quad 399$ & 3 & 2.22 & 1.064 & 0,01 \\
\hline $400-\quad 499$ & 5 & 3,70 & 2.326 & 0,02 \\
\hline $500-\quad 599$ & 3 & 2,22 & 1.577 & 0,01 \\
\hline $600-\quad 699$ & 1 & 0,74 & 600 & 0,01 \\
\hline $700-\quad 799$ & 33 & 24.45 & 23.620 & 0,22 \\
\hline $800-\quad 899$ & 11 & 8,15 & 9.020 & 0,08 \\
\hline $900-\quad 999$ & 16 & 11,85 & 14.440 & 0,13 \\
\hline $1.000-1.499$ & 28 & 20,75 & 32.806 & 0,31 \\
\hline $1.500-1.999$ & 15 & 11,11 & 23.866 & 0,22 \\
\hline $2.000-4.999$ & 8 & 5,93 & 27.434 & 0,26 \\
\hline $5.000-9.999$ & 4 & 2,96 & 25.782 & 0,24 \\
\hline $10.000-99.999$ & - & - & - & - \\
\hline \multirow[t]{2}{*}{+100.000} & $6(*)$ & 4,44 & 10.552 .518 & 98,48 \\
\hline & 135 & 100,00 & 10.715 .390 & 100,00 \\
\hline
\end{tabular}

(*) Se trata de grandes compradores que a nivel individual o en sociedades poseen tierras en Campoamor. Entre los primeros está en agricultor de Orihuela $\left(934,971 \mathrm{~m}^{2}\right)$ y un industrial de Elche $\left(400.000 \mathrm{~m}^{2}\right)$; pertenecen a los segundos las siguientes sociedades: Villas de Campoamor S. A. (502.000 $\left.\mathrm{m}^{2}\right)$, Inmobiliaria Colaboradores Bepasa S. A. $\left(160.000 \mathrm{~m}^{2}\right)$, Montepiedra S. A. $\left(1.450 .000 \mathrm{~m}^{2}\right)$ y la Sociedad Cooperativa Agrícola del Raiguero de Bonanza $\left(7.079 .324 \mathrm{~m}^{2}\right)$. 
La mayoría de compradores adquieren un solo lote. En La Cenia se dio la circunstancia de que el comprador tenía que presentar al dueño de la hacienda el proyecto de obra a realizar para su aprobación «a fin de procurar una perfecta armonización con la urbanización» que en dicho lugar se estaba realizando. Se trata de viviendas exentas unifamiliares, aspecto este también constatable en Campoamor. Si bien en las últimas décadas, coincidiendo con una mayor demanda y la actuación de grandes empresas inmobiliarias, se ha roto la uniformidad constructiva, aprovechando el suelo al máximo por su alto precio, mediante las torres de apartamentos y los bungalows. Los propietarios de esta finca no se mantuvieron al margen de este negocio especulativo y bien de forma individual o como accionistas de empresas mayores se dedican a la actividad inmobiliaria.

En cuanto al origen de los compradores destacan claramente los nacionales, que adquieren en Campoamor el $87,4 \%$ de los lotes y en La Cenia el $64,1 \%$

\section{CUADRO 3}

Procedencia de los compradores de parcelas en la urbanización La Zenia (1964-1986)

\begin{tabular}{lrrrr} 
Procedenciat & Compradores & \multicolumn{2}{c}{ Superficie $\mathrm{m}^{2}$} & $\%$ \\
Nacional & & & & \\
Murcia & 70 & 37.4 & 59.886 & 28.7 \\
Madrid & 35 & 18.7 & 44.891 & 21.5 \\
Orihuela & 21 & 11.2 & 15.614 & 7.5 \\
Bajo Segura & 15 & 8.0 & 29.826 & 14.3 \\
Prov. Alicante & 13 & 7.0 & 20.829 & 10.0 \\
Prov. Murcia & 16 & 8.6 & 14.980 & 7.2 \\
Otras Provincias & 14 & 7.5 & 17.142 & 8.2 \\
No consta & 3 & 1.6 & 5.474 & 2.6 \\
\hline Total & 187 & 100.0 & 208.642 & 100.0 \\
& & & & \\
Eviranjera & & & & \\
Inglaterra & 37 & 35.2 & 20.426 & 30.9 \\
Alemania & 31 & 29.6 & 17.316 & 26.3 \\
Suiza & 10 & 9.5 & 7.079 & 10.7 \\
Francia & 8 & 7.6 & 6.320 & 9.6 \\
Bélgica & 6 & 5.7 & 5.752 & 8.7 \\
Otros Paises & 13 & 12.4 & 9.071 & 13.8 \\
Total & 105 & 100.0 & 65.964 & 100.0
\end{tabular}


frente al $12,6 \%$ y $35,9 \%$ respectivamente de los extranjeros. Dentro de los primeros en ambas urbanizaciones destacan con mucho los madrileños, murcianos y oriolanos; siendo los extranjeros de diversa procedencia, predominando en La Cenia ingleses y alemanes y en Campoamor, junto a estos últimos, los franceses.

Referido a la actividad de los compradores hay que hacer referencia en los dos complejos residenciales analizados al gran número de industriales que aparecen como compradores, también destacan las profesiones liberales.

3b. Fincas agro-industriales. Pertenecen a este apartado dos fincas que están en poder de empresas que se han dedicado a la explotación agrícola. «Lo Romero» con $705 \mathrm{Ha}$. de tierra de campo, con cañadas y lomas cultivadas e incultas, con dos casas de labor, una bodega y una almazara.

A principios de este siglo, en 1908, este predio pertenecía a un cartagenero que la vendió a la empresa domiciliada en Valencia, Frutos Españoles S. A. en 1956. Esta empresa compró en esos mismos años otras fincas en la provincia de Murcia, en los términos de San Pedro del Pinatar y San Javier, que al agruparse con la existente en Orihuela constituyó una explotación de mayor dimensión, conocida genéricamente con el nombre de «Lo Romero», pero que incluye las antiguas haciendas de «Siete Higueras», así como las denominadas «Casa del Fraile», «Casa Nueva» y «La Pacheca» (estas tres últimas en la provincia de Murcia con una extensión de 207 Ha., lo que representa el $21,8 \%$ de la superficie total).

Esta entidad lleva a cabo la primera instalación de puesta en riego, al implantar torres de transformación de energía eléctrica para alimentar los motores de riego. En 1962 se hizo cargo de la explotación la compañía valenciana de cementos Portland, cancelando todas las hipotecas que pesaban sobre la tierra, con un valor próximo a 30 millones de pesetas.

En la actualidad, la finca cuenta con $550 \mathrm{Ha}$. en intensa explotación donde antaño existía un aprovechamiento de secano y monte. A los caudales que se obtenían de perforaciones, cada vez a mayor profundidad, hay que añadir las aguas del trasvase. Todo ello ha llevado a la creación de un gran complejo agro-industrial, donde los cítricos ocupan el 70\% de la zona cultivada y el resto está cubierto de perales y parral, así como $185.000 \mathrm{~m}^{2}$ bajo invernaderos.

La empresa cuenta con un centro administrativo, con un poblado para personal fijo (90 obreros)y albergues para el eventual, naves para maquinaria, taller de mantenimiento y una central hortofrutícola que alberga cinco cámaras frigoríficas y una de desverdización, donde se prepara la campaña exportadora. Parte de la cosecha se comercializa con marcas propias, que envían directamente a Francia y Alemania (20).

También es representativa la transformación operada en la hacienda «Lo Monte», ubicada en el partido de La Horadada, con una extensión de 355 Ha., 
plantada en una mínima parte de olivar y viña (el resto es tierra en blanco). La puesta en riego data de 1954 al solicitar su propietario al I. N. C. un préstamo para redimir definitivamente del secano como mínimo unas $200 \mathrm{Ha}$. Con posterioridad esta finca se agrupó con otras colindantes («Cueva Fuerte», «Lo Montanero», etc.) de iguales características hasta llegar a reunir 1.230 Ha. que se conocen genéricamente con el nombre de «Lo Monte». Esta propiedad se encuentra en un $70 \%$ puesta en regadío. Dicha transformación se ha realizado con ayuda de medieros y de una firma exportadora que se estableció a rento y que ha asumido directamente la explotación y en parte la comercialización. Entre los aprovechamientos agrícolas a destacar, cabe citar los frutales y la producción de hortalizas, tanto en invernaderos como a la intemperie, resaltando las tomateras (21).

En esta finca se ha producido una segregación de 47 Ha. vendidas en 1972 a Continental Costablanca S. A., con la concesión de un caudal de agua de hasta $500 \mathrm{~m}^{3} /$ día procedentes de los pozos que hay en la finca. Son interesantes las condiciones descritas en la escritura de venta para el uso de este agua que es: para utilizarla en la finca vendida, sin que pueda ser traspasada a terceros; si existen sobrantes del caudal diario, el comprador se comprometía a remitir al vendedor el agua a una balsa existente en la hacienda «La Vereda», y si éste la vende a un tercero tiene que darle al anterior el $20 \%$ del precio de venta; el agua se abona al vendedor al mismo precio que marque el ayuntamiento de San Pedro del Pinatar; la concesión no tiene límite en el tiempo, salvo que se agoten los pozos.

3c. Fincas agrícolas. Son más numerosas las haciendas que en este período de tiempo han mantenido una dedicación eminentemente agrícola, vinculadas unas veces a sus antiguos aprovechamientos en espera de los aportes hídricos necesarios, y solamente en el mejor de los casos han acometido transformaciones. Muchas veces el adquirir estas tierras de secano y su no transformación indican un intento de prestigio social.

Este proceso lo hemos seguido en dos fincas, la primera se llama «Dehesa de Roca», que constituye un predio de $516 \mathrm{Ha}$. que ha estado vinculado tradicionalmente a la familia nobiliaria de los Roca de Togores. Tras la Guerra Civil esta propiedad se incluía entre los patrimonios del duque de Pino Hermoso por matrimonio con $D^{\mathrm{a}}$. Carmen Fernández Villaverde y Roca de Togores. Dicho propietario era uno de los grandes terratenientes que quedaban en la comarca al detentar antiguos predios de origen señorial, entre ellos cabe citar Benejúzar (22).

Un agricultor adquirió esta propiedad en 1940, entonces solicitó un préstamo del I. N. C. para transformar en regadío $60 \mathrm{Ha}$. de la finca mediante un pozo que suministraba 50 1/s. Dicho comprador inició rápidamente un proceso de venta en pequeños lotes, que comenzó en 1942 y llega hasta 1983, vendiendo 118 lotes con una superficie de 184,8101 Ha., lo que representa el $35,8 \%$ de la finca. 
Los lotes vendidos están comprendidos entre menos de 0,24 Ha. hasta más de 10 Ha. Las fincas con menos de $1 \mathrm{Ha}$. son las que cuentan con más ventajas para la implantación del regadío. Casi la totalidad de los compradores poseen un lote. Hay que destacar que todos los compradores pertenecen a las provincias de Murcia y Alicante, sobresaliendo los de Murcia capital y Orihuela. Se trata por lo general de personas vinculadas a la tierra, si bien no se excluyen compradores de otras actividades.

Por lo que respecta a la segunda finca llamada «Vista Alegre», hemos de decir que posee un proceso similar al anterior pero de menor magnitud. Tiene $81 \mathrm{Ha}$., y está ubicada en el partido de La Murada. De todas las fincas analizadas es esta la que cuenta con un disfrute de agua más antiguo, pues se incluye dentro de la infraestructura de regadío creada por Riegos de Levante S. A. a principios del siglo XX al disfrutar de los caudales sobrantes del Segura, que por su carácter de aguas excedentarias no se pudo transformar totalmente el agro, y recientemente de los caudales del Tajo, que han posibilitado el proceso de transformación hacia una agricultura intensiva. Aspecto que ha ido acompañado de una fragmentación de la explotación, son 11 segregaciones realizadas, cinco de ellas por particiones hereditarias con una superficie unitaria de casi 12 Ha., y el resto ha sido vendido a particulares desde la década de los setenta con superficies que van desde 1,5 Ha. hasta $8 \mathrm{Ha}$.

\section{Sociedades que operan en el campo}

Tras la visión efectuada se observa que las grandes haciendas han sido objeto de atracción para las grandes empresas, se trata en unos casos de sociedades de orientación exclusivamente agraria, en otros de entidades de ahorro que han invertido en la puesta en valor de estos predios, siendo las auténticas protagonistas del cambio paisajístico realizado pues la puesta en explotación ha requerido grandes inversiones para la transformación en regadío, que se ha realizado, unas veces para su venta, obteniendo sustanciosas plusvalías. Se analizan por último algunas de las sociedades que hemos detectado en el campo oriolano además de las ya indicadas, que son fiel reflejo de la importancia que ha cobrado la agricultura capitalista en este área en las dos últimas décadas. La casuística de formación de las empresas es muy variada.

En algunos casos se trata de adquisiciones de tierra por parte de las empresas ya constituidas y que invierten con intenciones lucrativas e industriales, este es el caso más corriente, y consiste en adquirir fincas de secano y proceder a su puesta en riego. Este cambio de uso y propietario se ejemplifica con el proceso de la hacienda «La Tahona» de $37 \mathrm{Ha}$., que fue adquirida por la compañía murciana Sarqui S. A., o parte de la finca «El Garbanzuelo» de 97 Ha., vendida a la compañía alicantina Exfinag S. A. (23); o la acumulación de parcelas de distintas fincas como la efectuada por la Compañía Mercantil Maderas de Cantabria S. A., de Madrid, que acumula 117 Ha., de las fincas «San Luis», «Santa Catalina» $\mathrm{y}$ 
«El Chaparral», con un capital social de 5 millones distribuido en 10.000 acciones de 500 pesetas nominales, compra íntegramente la Caja Territorial de Madrid S. A. en 1979, la cual ha llevado a cabo la urbanización de este espacio que rodea las Salinas de La Mata (24).

Otra forma de llegar a la formación de una gran empresa es la aportación por parte de un propietario de la tierra como capital social de la misma adquiriendo todas las acciones en un primer momento, aunque después puedan acceder otros socios, este es el caso de las fincas del litoral que compaginan aprovechamientos agrícolas con los derivados del turismo. Como hacienda representativa encontramos la de «Campoamor», donde el titular emprendió la urbanización del predio mediante la segregación de pequeños lotes para la construcción de chalets, con posterioridad se asocia con otras personas y crean sociedades para explotaciones diversas, tanto actividades hoteleras como inmobiliarias, entre ellas cabe citar: Montepiedra S.A., Gran Hotel Campoamor S.A. y Villas de Campoamor S.A.

Un tercer paso es aquel en el que el dueño de la tierra la cede por su valor al capital social y al mismo tiempo otros accionistas invierten dinero, esta inversión puede llegar por una doble vía: de empresas ya formadas o de accionistas individuales, que forman parte de la sociedad, casi siempre minoritariamente. Dentro de la primera vía está la sociedad Balcones S.A (BALCOSA), empresa formada por el dueño de la tierra, en unión de la Caja de Ahorros y Socorro y Monte de Piedad Nuestra Señora de Monserrate de Orihuela, además de otros socios particulares que forman en 1968 la asociación, y que va a realizar la urbanización de igual nombre (25). Dentro de la segunda vía queda perfectamente definida la entidad Explotaciones Agrícolas Rebate S.A., creada inicialmente en 1983 con la aportación de la tierra por parte de un propietario, adquiriendo por ello más de la mitad de las acciones, y la entrega en metálico del resto del capital por parte de tres individuos de nacionalidad belga. En la actualidad tras nuevas aportaciones de tierra, la empresa reúne algo más de 700 Ha. de cultivo intensivo (26).

\section{NOTAS}

1. CANALES MARTíNEZ, G., «Primer intento de transformación en el secano del Bajo Segura: la Ley de 3 de junio de 1868 sobre Colonias Agrícolas», Ministerio de Agricultura (en prensa).

18. RAMOS VIDAL, J. A., Demografia, economía y sociedad en la comarca del Bajo Segura durante el siglo XVIII, Orihuela, C.A.A.M., 1980, p. 78.

19. VERA REBOLLO, J.F., Tradición y cambio en el campo del Bajo Segura, Alicante, Diputación Provincial, 1984, p. 95. 
20. COSTA MAS, J., «Cambio de estructuras agrarias al sur de Alicante (aproximación al mercado de la tierra en el Bajo Segura)», La propiedad de la tierra en España, Alicante, Departamento de Geografía, Facultad de Filosofía y Letras, 1981, p. 433.

21. COSTA MAS. J., «La agricultura de los cosecheros-exportadores de tomate de invierno en Alacant», Investigaciones Geográficas, $\mathrm{n}^{\circ}$. 2, Alicante, Instituto Universitario de Geografía, 1984, p. 75.

22. GIL OLCINA, A., La propiedad señorial en tierras valencianas, Valencia, del Cenia al Segura, 1979, p. 164.

23. Registro de la Propiedad de Orihuela (en adelante R. P. O.). Libro de inscripciones del municipio de Orihuela, t. 866, f. 169.

24. R. P. O., Libro de inscripciones del municipio de Orihuela, t. 734, f. 188.

25. R. P. O., Libro de inscripciones del municipio de Orihuela, t. 604, f. 177.

26. R. P. O., Libro de inscripciones del municipio de Orihuela, t. 1.110, f. 31. 